\title{
Serum Leptin Concentration is Correlated to Insulin Resistance in Polycystic Ovary Syndrome (PCOS) Patients
}

\author{
Arsyi Adliah Anwar ${ }^{1}$, Nusratuddin Abdullah ${ }^{1}$, Andi Nursanty Padjalangi ${ }^{1}$, Firdaus Hamid ${ }^{2}$, \\ Nasrudin Andi Mappeware ${ }^{1}$, Efendi Lukas ${ }^{1}$ \\ ${ }^{1}$ Obstetrics and Gynecology Department, Faculty of Medicine, Universitas Hasanuddin, Makassar, Indonesia \\ ${ }^{2}$ Faculty of Medicine, Universitas Hasanuddin, Makassar, Indonesia
}

Background: Leptin resistance which leads to excessive circulating leptin levels is thought to affect ovarian function. This study aimed to study the correlation between serum leptin levels with insulin resistance in patients with polycystic ovary syndrome.

Materials and Methods: This cross-sectional study was undertaken in several teaching hospitals in Makassar, Indonesia. We included patients diagnosed with polycystic ovary syndrome (PCOS) aged 18-40 years old. Serum leptin levels were examined in all eligible subjects using the enzyme-linked immunosorbent assay (ELISA) method. The results obtained were further analyzed statistically.

Results: Approximately 53 PCOS subjects were included in this study, 25 subjects with insulin resistance and 28 subjects without insulin resistance. After examining serum leptin levels, we found that leptin is directly proportional to insulin resistance $(p<0.001)$. We even found a strong positive correlation between serum leptin levels with homeostatic model assessment for insulin resistance (HOMA-IR) levels ( $r=0.659 ; p<0.001)$. Leptin was found to be independent of HOMA-IR, not influenced by confounding factors such as body mass index (BMI) $(p=0.090)$.

Conclusion: There was a significant correlation between serum leptin levels and HOMA-IR values in PCOS patients. This correlation was found to be significant regardless of patient's BMI, therefore is considered to have a direct effect on insulin resistance in PCOS.

Keywords: polycystic ovary syndrome, leptin, insulin resistance, HOMA-IR

\section{Introduction}

Polycystic ovary syndrome (PCOS) is one of the most common endocrine metabolic disorders, with an incidence rate of about $6-10 \%$, among women of reproductive age. ${ }^{1}$
Existing studies suggest that insulin resistance is closely linked with PCOS and is thought to be the center of all manifestations in this syndrome. Insulin resistance occurs in the majority $(65-90 \%)$ of obese patients and $25-45 \%$ in non-obese patients. Insulin resistance causes defects in the

Date of submission: March 6, 2021

Last Revised: May 7, 2021

Accepted for publication: May 11, 2021

Corresponding Author:

Arsyi Adliah Anwar

Obstetrics and Gynecology Department

Faculty of Medicine, Universitas Hasanuddin

Jl. Perintis Kemerdekaan Km. 10

Makassar 902425, South Sulawesi, Indonesia

e-mail: arsyiadliahanwar@gmail.com

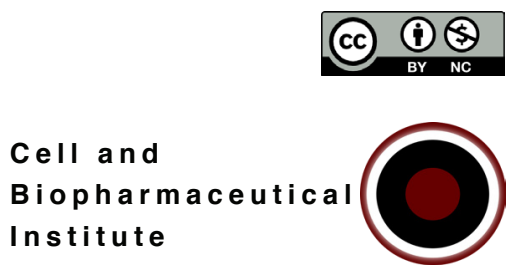


receptor or post-receptor area on peripheral cells (skeletal muscle, fibroblasts, adipose) so that it fails to respond to the presence of insulin. Hyperinsulinemia in PCOS then occurs due to negative feedback from the state of insulin resistance. Two main effects caused by hyperinsulinemia are its effects on vascular endothelial cells and ovarian theca cells. ${ }^{2}$

Insulin stimulates the biosynthesis of ovarian steroid hormones, especially ovarian androgens, in the same way that luteinizing hormone (LH) does. Insulin lowers the liver's production of sex-hormone-binding globulin (SHBG), resulting in a rise of free androgens. Therefore, these two pathways end with ovarian theca cell stimulation and increased ovarian androgen production, resulting in impaired folliculogenesis, menstrual cycle disorders, and chronic oligo or anovulation. ${ }^{3,4}$

Despite advanced research on this matter, the mechanism of insulin resistance in patients with PCOS is still highly debatable. Latest discussions have mentioned leptin, an appetite suppressant hormone secreted by adipose tissue having a role in this mechanism. ${ }^{5}$ Leptin receptors are found on pancreatic beta cells producing insulin so that leptin directly is assumed to play a role in regulating insulin secretion. ${ }^{6}$ Leptin also promotes steroidogenesis in granulosa and theca cells. ${ }^{7}$

A recent study found that in a state where there is interference with leptin signal in the hypothalamus and liver of humans and obese mice, a similar condition is not found in the kidneys and heart. It indicates a specific response from each organ. This study found for the first time the condition of leptin resistance in the ovaries of obese rats which then experienced impaired follicle growth, ovulation, and oocyte quality. ${ }^{8}$

Leptin resistance which leads to excessive circulating leptin levels ${ }^{9}$ is believed to affect ovarian function. Several mechanisms are considered as the cause. It also appears to be associated with suboptimal folliculogenesis. Overstimulation of leptin, both direct or indirectly through interactions with insulin, insulin-like growth factor (IGF), and other factors, disrupting hormonal conditions and creating a suboptimal environment. If leptin does play a role in ovarian function normally, leptin tolerance may explain the correlation between high leptin levels and low follicular production. Leptin inactivity in theca cells can reduce perifollicular vascularization resulting in intrafollicular hypoxia. This hypoxic process will interfere with glucose oxidation and adenosine triphosphate (ATP) production needed in the follicle maturation process. ${ }^{10}$
Based on these interesting findings, we aimed to study the correlation between serum leptin levels with insulin resistance in patients with polycystic ovary syndrome.

\section{Materials and methods}

\section{Subject Recruitment and Ethical Statements}

This cross-sectional study was undertaken in several teaching hospitals in Makassar, Indonesia. We included patients diagnosed with PCOS aged 18-40 years old. We exclude patients who were pregnant or breastfeeding, had an underlying medical condition which may cause hyperandrogenism or may interfere with the evaluation of this study, had impaired renal or hepatic functions, currently following a weight loss program or undergoing laparoscopic ovarian diathermy or an in vitro fertilization program, receiving medication that may affect insulin sensitivity and reproductive function within three months before this study, or participating in other clinical researches outside this study.

This study was approved by the Ethics Committee of Faculty of Medicine, Universitas Hasanuddin (No. 838/ UN4.6.4.5.31/PP36/2019). Before participating in the study, all participants already signed the written informed consents.

\section{History-taking and Physical Examination}

History taking was performed on subjects who fulfilled the criteria of the study and also provided their consent beforehand. The data was recorded on a questionnaire sheet. Physical examinations performed are included: an examination of vital signs, body weight, waist circumference, and the Modified Ferriman-Gallwey score assessment, a hirsutism scoring system focused on the quantification of body hair in nine different areas of the body, with scores ranging from 0 to 4 ( 0 : no hair development; 1 : vellus hair development; 2: terminal hair development less than 10; 3 : limited, terminal hair development more than 10; 4: frankly virile). A total score of $>8$ indicates hirsutism, with a score of 8-15 indicating mild hirsutism, 16-25 for moderate, and $>25$ for severe. ${ }^{11}$

\section{Serum Leptin Examination}

Serum leptin levels were examined in all eligible subjects using the enzyme-linked immunosorbent assay (ELISA) method. The ELISA kit we used was the Leptin ELISA manufactured by DBC-Diagnostics Biochem Canada Inc. 
(London, Ontario). The principle of this test follows a typical two-step capture or 'sandwich' type assay. Approximately 4-5 $\mathrm{ml}$ of blood was collected from our patients and later centrifuged in order to collect the serum sample to be used during the assay procedure.

\section{Statistical Analysis}

The results obtained were further analyzed with SPSS version 25.0 (IBM Corporation, Armonk, NY, USA) using chi-square and fisher's exact test for all categorical variables. Pearson's and Spearman's correlation test was carried out to assess the correlation between variables. A degree of significance or $p$-value $<0.05$ is considered significant. Furthermore, independent variables interaction were assessed by using two-way ANOVA.

\section{Results}

Approximately 53 PCOS subjects were included in this study. The diagnosis of polycystic ovary syndrome was made based on history taking, physical examination, and additional investigations that were found necessary. To confirm insulin resistance status, homeostatic model assessment for insulin resistance (HOMA-IR) levels were examined. HOMA-IR levels of 2 and above were considered insulin resistant and a HOMA-IR below 2 were considered not insulin resistant. We later found 25 patients were insulin resistant while 28 patients were not insulin resistant. After grouping subjects into their respective groups, all subjects were examined for leptin levels by the ELISA method. The characteristics of the research sample can be seen in Table 1 as follows.

Table 1. Subject's characteristics.

\begin{tabular}{|c|c|c|c|}
\hline Variables & $\begin{array}{l}\text { Insulin Resistant } \\
\text { (HOMA-IR } \geq 2 \text { ) }\end{array}$ & $\begin{array}{c}\text { Not Insulin Resistant } \\
\text { (HOMA-IR <2) }\end{array}$ & $p$-value \\
\hline \multicolumn{4}{|l|}{ Age } \\
\hline$<30$ years old & $21(50 \%)$ & $21(50 \%)$ & $0.420^{a}$ \\
\hline$\geq 30$ years old & $4(36.4 \%)$ & $7(63.6 \%)$ & \\
\hline \multicolumn{4}{|l|}{ Menstrual Cycle } \\
\hline Normal & $1(33.3 \%)$ & $2(66.7 \%)$ & $0.067^{b}$ \\
\hline Oligomenorrhea & $18(41.9 \%)$ & $25(58.1 \%)$ & \\
\hline Amenorrhea & $6(85.7 \%)$ & $1(14.3 \%)$ & \\
\hline \multicolumn{4}{|c|}{ Modified Ferriman-Gallwey Score (mFG) } \\
\hline Mild (Score $8-\leq 15$ ) & $25(47.2 \%)$ & $28(52.8 \%)$ & $*$ \\
\hline Moderate (Score 16-25) & 0 & 0 & \\
\hline Severe $($ Score $\geq 26$ ) & 0 & 0 & \\
\hline \multicolumn{4}{|l|}{ BMI (Body Mass Index) } \\
\hline Normal (18.5-24.9) & $6(33.3 \%)$ & $12(66.7 \%)$ & NA \\
\hline Overweight (25-29.9) & $11(40.7 \%)$ & $16(59.3 \%)$ & \\
\hline Obese $(\geq 30)$ & $8(100 \%)$ & 0 & \\
\hline \multicolumn{4}{|l|}{ Leptin Serum Levels } \\
\hline$<37.94 \mathrm{ng} / \mathrm{mL}$ & $5(17.2 \%)$ & $24(82.8 \%)$ & $<0.001^{a}$ \\
\hline$\geq 37.94 \mathrm{ng} / \mathrm{mL}$ & $20(83.3 \%)$ & $4(16.7 \%)$ & \\
\hline
\end{tabular}

${ }^{a}$ Chi-sqaure, significant if $p$-value $<0.05$. *no statistical analysis was performed because the modified Ferriman-Gallwey score in this case was constant. 
We discovered that variables such as age $(p=0.420)$ and menstrual cycle characteristics $(p=0.067)$ are not correlated to insulin resistance in these patients (Table 1). After examining serum leptin levels, we found that as leptin increases, the HOMA-IR value will increase, and vice versa (Figure 1). In other words, leptin is directly proportional to insulin resistance $(p<0.001)$.

We even found a strong positive correlation between serum leptin levels with HOMA-IR levels ( $\mathrm{r}=0.659$; $p<0.001)$. Furthermore, to ensure that the results obtained were not influenced or not caused by an interaction between leptin and other confounding factors, further analysis was carried out to analyze the relationship between body mass index (BMI) and HOMA-IR followed by analyzing the interaction between BMI variables and leptin levels with HOMA-IR using two-way ANOVA and found that leptin is associated independently with HOMA-IR and is not affected by other confounding factors such as body mass index $(p=0.090)$.

\section{Discussion}

Leptin is an adipokine produced by adipose tissue and is thought to have any involvement in the incidence of obesity, insulin resistance, and PCOS. This study aimed to assess the relationship between leptin levels and insulin resistance in patients with PCOS. The correlation between these two variables is the main parameter studied. A total of 53 subjects were diagnosed with polycystic ovary syndrome consisting of 25 subjects with insulin resistance confirmed by a HOMA-IR of 2 and above while 28 subjects were not insulin resistant, confirmed by a HOMA-IR less than 2 .

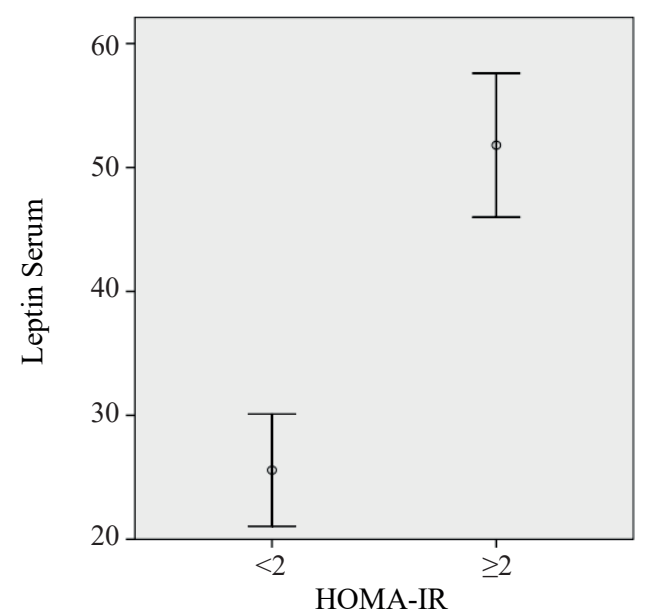

Figure 1. Serum leptin levels based on HOMA-IR values.
Our data showed that as leptin increases, HOMAIR values increase as well, and vice versa. In other words, leptin is directly proportional to insulin resistance. This finding was statistically significant with a strong positive correlation between leptin and HOMA-IR. This result was the most interesting finding of this study. Our findings were consistent with previous studies which stated that insulin acutely increases plasma leptin and reduces adiponectin making them directly proportional. It suggests the role of hyperinsulinemia in cases of insulin resistance, which is associated with increased adipocyte inflammatory factors in patients with PCOS. ${ }^{12}$

Leptin was independent of HOMA-IR, not influenced by confounding factors such as BMI. This finding is consistent with previous study which stated that leptin was significantly correlated with HOMA-IR regardless of the patient's $\mathrm{BMI}^{13}$, considering that $\mathrm{BMI}$ is also a factor that is often associated with insulin resistance. This finding was due to leptin having a direct effect on pancreatic beta cells to inhibit insulin secretion. Leptin can induce pro-inflammatory cytokines such as C-reactive protein and interleukin-6 which can cause pancreatic beta-cell apoptosis. ${ }^{14}$ Another study found that hyperplasia in pancreatic islet cells of mice that are deficient in leptin signals due to defects in leptin receptors and as a consequence of excessive insulin production. ${ }^{15}$

Furthermore, although most treatments of obesity achieve modest reductions in weight and improvements in the PCOS phenotype, encouraging weight loss in the obese patient remains one of the front-line therapies plus life style modification is also very encouraging. However, new studies are required to identify the best interventions, and the role of lifestyle therapies in women of normal weight with PCOS is uncertain. ${ }^{16}$ The prevalence of obesity in developed countries has doubled in the past two decades. ${ }^{17}$ Some studies suggest that total weight loss and the loss of a crucial amount of abdominal fat are important treatment strategies. The reproductive and metabolic benefits resulting from weight loss are presumed to result from a reduction in circulating insulin. ${ }^{18}$ Such dietary restrictions when combined with moderate energy expenditure, are described as being most likely to produce sustained weight loss and favorable endocrine and reproductive outcomes among women with PCOS. ${ }^{19}$ Lifestyle modification consists of a multifaceted approach of dietary, exercise and behaviour therapies, which aim to educate an individual's principles and techniques to achieve dietary and exercise goals. ${ }^{20}$ 
There are several limitations in this study. First, there are still several confounding factors that were not able to be controlled in our analysis. Besides, the very complex pathophysiology of PCOS leads to the need for numerous parameters needed to be taken into consideration and analyzed which were not the focus of this study. However, this study has controlled for confounding variables such as obesity which also affects the condition of insulin resistance in addition to leptin levels, so that the results of the strong significant correlation between leptin levels and insulin resistance in PCOS patients obtained in this study were fundamentally logical.

\section{Conclusion}

In conclusion, there was a significant correlation between serum leptin levels and HOMA-IR values in PCOS patients. Leptin and HOMA-IR levels were found to be directly proportional. This correlation was found to be significant regardless of the patient's BMI. Therefore, leptin is considered to have a direct effect on insulin resistance in PCOS. Further multicenter research on the relationship of insulin resistance in patients with polycystic ovary syndrome with variables other than leptin which are also considered potential contributors are of utmost importance to improve current understanding of the concept of insulin resistance in patients with polycystic ovary syndrome.

\section{References}

1. Azziz R, Carmina E, Chen Z, Dunaif A, Laven JSE, Legro RS, et al. Polycystic ovary syndrome. Nat Rev Dis Prim. 2016; 2: 16057. doi: 10.1038/nrdp.2016.57.

2. Schachter M, Raziel A, Strassburger D, Rotem C, Ron-El R, Friedler $\mathrm{S}$. Prospective, randomized trial of metformin and vitamins for the reduction of plasma homocysteine in insulin-resistant polycystic ovary syndrome. Fertil Steril. 2007; 88(1): 227-30.

3. Diamanti-Kandarakis E. Insulin resistance in PCOS. Endocrine. 2006; 30(1): 13-8.

4. McLuskie I, Newth A. New diagnosis of polycystic ovary syndrome. BMJ. 2017; 356: i6456. doi: 10.1136/bmj.i6456.

5. Ramos-Lobo AM, Donato J Jr. The role of leptin in health and disease. Temperature (Austin). 2017; 4(3): 258-291.

6. Azziz R. Definition, diagnosis, and epidemiology of the polycystic ovary syndrome. In: The Polycystic Ovary Syndrome: Current
Concepts on Pathogenesis and Clinical Care. New York: Springer; 2007. p. 1-15.

7. de Medeiros SF, Rodgers RJ, Norman RJ. Adipocyte and steroidogenic cell cross-talk in polycystic ovary syndrome. Hum Reprod Update. 2021; 27(4): 771-96.

8. Wołodko K, Walewska E, Adamowski M, Castillo-Fernandez J, Kelsey G, Galvão A. Leptin resistance in the ovary of obese mice is associated with profound changes in the transcriptome of cumulus cells. Cell Physiol Biochem. 2020; 54(3): 417-37.

9. Lukito AA, Bakri S, Kabo P, Wijaya A. The mechanism of coronary artery calcification in centrally obese non-diabetic men: study on the interaction of leptin, free leptin index, adiponectin, hs-C reactive protein, bone morphogenetic protein-2 and matrix gla protein. Mol Cell Biomed Sci. 2020;4(2): 88-93.

10. Henson MC, Castracane VD, Edwards DE. Leptin: from satiety signal to reproductive regulator. In: Leptin and Reproduction. New York: Springer; 2003. p. 3-11.

11. Parlak M, Parlak AE, Toslak IE. Etiologic factors and clinical characteristics of Turkish adolescent girls diagnosed with hirsutism. J Clin Anal Med. 2016; 7(2): 205-9.

12. El-Gharib MN, Badawy TE. Correlation between insulin, leptin and polycystic ovary syndrome. J Basic Clin Reprod Sci. 2014; 3(1): 49-53.

13. Huang KC, Lin RCY, Kormas N, Lee LT, Chen CY, Gill TP, et al. Plasma leptin is associated with insulin resistance independent of age, body mass index, fat mass, lipids, and pubertal development in nondiabetic adolescents. Int J Obes. 2004; 28(4): 470-5.

14. Moonishaa TM, Nanda SK, Shamraj M, Sivaa R, Sivakumar P, Ravichandran K. Evaluation of leptin as a marker of insulin resistance in type 2 diabetes mellitus. Int $\mathrm{J}$ Appl basic Med Res. 2017; 7(3): 176-80.

15. Morioka T, Asilmaz E, Hu J, Dishinger JF, Kurpad AJ, Elias CF, et al. Disruption of leptin receptor expression in the pancreas directly affects $\beta$ cell growth and function in mice. J Clin Invest. 2007; 117(10): 2860-8.

16. Shahid M, Naveed M, Khalid S, Atif M, Tufail T, Shahid MZ, et al. Nutritional and lifestyle modifications of polycystic ovary syndrome (pcos) in obesity. Int J Biosci. 2019; 5(6): 259-72.

17. Hoeger KM. Role of lifestyle modification in the management of polycystic ovary syndrome. Best Pract Res Clin Endocrinol Metab. 2006; 20(2): 293-310.

18. Norman RJ, Davies MJ, Lord J, Moran LJ. The role of lifestyle modification in polycystic ovary syndrome. Trends Endocrinol Metab. 2002; 13(6): 251-7.

19. Arentz S, Smith CA, Abbott J, Bensoussan A. Perceptions and experiences of lifestyle interventions in women with polycystic ovary syndrome (PCOS), as a management strategy for symptoms of PCOS. BMC Womens Health. 2021; 21(1): 107. doi: 10.1186/ s12905-021-01252-1.

20. Moran LJ, Brinkworth G, Noakes M, Norman RJ. Effects of lifestyle modification in polycystic ovarian syndrome. Reprod Biomed Online. 2006; 12(5): 569-78. 